For Internal Distribution Only

\author{
Accelerator Division \\ Alternating Gradient Synchrotron Department \\ BROOKHAVEN NATIONAL LABORATORY \\ Upton, New York 11973
}

Accelerator Division

Technical Note

AGS/AD/Tech. Note No. 449

CALCULATION OF INCOHERENT SP ACE CHARGE TUNE SPREAD

S. Y. Zhang, T. Roser and W. T. Weng

October 11, 1996 


\title{
Calculation of Incoherent Space Charge Tune Spread
}

\author{
S.Y. Zhang, T. Roser and W.T. Weng
}

\begin{abstract}
Literatures on incoherent space charge tune spread in a synchrotron have been surveyed. Unified calculations are adopted based on simplified models.
\end{abstract}




\section{Introduction}

It is not unusual to find some discrepancies in calculations of the incoherent space charge tune spread for a synchrotron. These discrepancies, sometimes large, do not necessarily imply errors, because oftentimes different criteria are used. Although understandable, it is not convenient for the readers. It is also well known that the beam size, the transverse emittance, and the bunching factor used in the calculations, if not clearly stated, may have different definitions and implications. In this note, we give a brief survey and try to address an unified calculation, based on a simplified model.

\section{Simplified Model}

In $[1,2,3]$, the vertical space charge incoherent tune of the coasting beam is defined as,

$$
\Delta \nu_{i n c}^{V}=\frac{-N R r_{0}}{\pi \nu_{0} \beta^{2} \gamma}\left(\beta^{2} \frac{\epsilon_{1}^{V}}{b_{V}^{2}}+\beta^{2} \frac{\epsilon_{2}^{V}}{g^{2}}+\frac{1}{\gamma^{2} a_{V}\left(a_{H}+a_{V}\right)}\right)
$$

where $N$ is the total number of particles, $R$ is the machine radius, $r_{0}$ is the classical radius of proton, $1.535 \times 10^{-18} \mathrm{~m}, \nu_{0}$ is the betatron tune with zero beam current, and $b_{V}$ is the average half chamber height in vertical direction, $g$ is the half pole gap, $a_{H}$ and $a_{V}$ are the average radii of the beam in the horizontal and vertical directions, respectively, and $\epsilon_{\mathbf{1}}$ and $\epsilon_{2}$ are the Laslett incoherent electric and magnetic coefficients, respectively.

For horizontal incoherent tune shift, we have

$$
\Delta \nu_{i n c}^{H}=\frac{-N R r_{0}}{\pi \nu_{0} \beta^{2} \gamma}\left(\beta^{2} \frac{\epsilon_{1}^{H}}{b_{V}^{2}}+\beta^{2} \frac{\epsilon_{2}^{H}}{g^{2}}+\frac{1}{\gamma^{2} a_{H}\left(a_{V}+a_{H}\right)}\right)
$$

where

$$
\epsilon_{1,2}^{H}=-\epsilon_{1,2}^{V}
$$

For the simplified model, we consider circular chamber, which gives rise to,

$$
\epsilon_{1}^{V, H}=\epsilon_{2}^{V, H}=0
$$

We also consider first the symmetric beams. Thus, the incoherent space charge tune spread can be calculated from (1) by,

$$
\Delta \nu_{i n c}=\frac{-N R r_{0}}{2 \pi \nu_{0} \beta^{2} \gamma^{3} a^{2}}
$$




\section{Coasting Beam}

Using (5) to calculate the coasting beam space charge tune spread, the beam radius a needs to be clearly defined. In Laslett's work [4], uniform particle distribution is considered. For a Gaussian distribution, Zotter [1] found that one has to set,

$$
a=\sqrt{2} \sigma
$$

where $\sigma$ is the rms beam radius, or the standard deviation in a Gaussian distribution.

Hofmann in [3] also used the relation (6) for a Gaussian.

In Chao [5], the tune shift is written,

$$
\Delta \nu_{i n c}=\frac{-\lambda r_{0} R^{2}}{\nu_{0} a^{2} \beta^{2} \gamma^{3}}
$$

where the average line density $\lambda$ is defined as,

$$
\lambda=\frac{N}{2 \pi R}
$$

Substituting (8) into (7), we get (5). Note that the beam radius $a$ in [5] is actually defined in

$$
\epsilon_{r m s}=\frac{a^{2}}{2 \bar{\beta}}
$$

where $\epsilon_{r m s}$ is the $r m s$ emittance, $\bar{\beta}$ is the average beta function. Therefore, for a Gaussian distribution this beam radius is the same as that in (6).

We note that if one uses (6) the elaboration represented by multiplying a form factor accounting for the transverse particle distribution, such as in [6], will not be needed.

One may calculate the incoherent space charge tune spread by using the normalized beam emittance as,

$$
\Delta \nu_{i n c}=\frac{-3 N r_{0}}{2 \pi \beta \gamma^{2} \epsilon_{N, 95 \%}}
$$

Substituting

$$
\epsilon_{N, 95 \%}=\frac{\beta \gamma}{\bar{\beta}(s)} a_{95 \%}^{2}
$$

with

$$
\bar{\beta}(s) \approx \frac{R}{\nu_{0}}
$$

and

$$
a_{95 \%}=\sqrt{6} \sigma=\sqrt{3} a
$$

the equation (10) becomes the same as (5). 


\section{Bunched Beam}

In $[1,2,7]$, the tune spread for bunched beams is modified from (5) as,

$$
\Delta \nu_{i n c}=\frac{-N R r_{0}}{2 \pi \nu_{0} B_{f} \beta^{2} \gamma^{3} a^{2}}
$$

or in terms of the emittance,

$$
\Delta \nu_{i n c}=\frac{-3 N r_{0}}{2 \pi B_{f} \beta \gamma^{2} \epsilon_{N, 95 \%}}
$$

The bunching factor $B_{f}$ is defined in [7] as,

$$
B_{f}=\frac{I_{0}}{I_{p}}=\frac{4 \sigma_{z} M}{2 \pi R S}
$$

where $I_{0}$ and $I_{p}$ are the average and peak beam current, respectively, $\sigma_{z}$ is the rms bunch length in meter, $M$ is the number of the bunch, and $S=1.5$ for parabolic and $S=2 \sqrt{2 / \pi}=$ 1.596 for Gaussian line densities, respectively.

It should be noticed that in many papers, especially in those concerning the beam longitudinal instabilities, such as in [8], the bunching factor is defined in a way as,

$$
B=\frac{4 \sigma_{z} M}{2 \pi R}
$$

which is convenient in terms of the spectrum analysis of the beam line density.

Using (5) or (10), the calculated tune spread for coasting beams is the maximum one, which applies to the particles located in the center of the phase space distribution. Note that these particles have small betatron oscillation amplitudes. For the bunched beams, with the bunching factor $B_{f}$, the beam peak current is used. This implies that the tune spread calculated using (14) or (15) further applies to the particles located in the bunch azimuthal center. Since those particles will certainly migrate away from the bunch center and in turn experience a smaller tune shift, often the maximum tune spread can across the half integer or integer without causing significant envelope oscillation and emittance blow-up. In other words, the collective modes, rather than the individual particles, are relevant to the emittance growth and beam loss owing to the stopband crossings.

To avoid ambiguity in the calculation, we may use the normalized $r m s$ beam emittance $\epsilon_{N, r m s}$, and also the rms bunch length in meter, rather than the bunching factor. Thus, using the relation

$$
\epsilon_{N, 95 \%}=6 \epsilon_{N, r m s}
$$

the equation (15) becomes,

$$
\Delta \nu_{i n c}=\frac{-N r_{0} R S}{8 \sigma_{z} M \beta \gamma^{2} \epsilon_{N, r m s}}
$$

where $N$ is the total number of the particles in the ring. 


\section{Asymmetric Beam}

For a circular chamber, the tune spread for the asymmetric beam can be calculated from the equations (1) and (4). Using the equation (6) and the following relation,

$$
\epsilon_{N, r m s}=\frac{\nu_{0}}{R} \beta \gamma \sigma^{2}
$$

we get, for example, the vertical tune spread as,

$$
\Delta \nu_{i n c}^{V}=\frac{-N r_{0} R S}{4 \sigma_{z} M \beta \gamma^{2} \epsilon_{N, r m s}^{V}\left(1+\sqrt{\epsilon_{N, r m s}^{H} / \epsilon_{N, r m s}^{V}}\right)}
$$

\section{Comments}

- The maximum space charge tune spread calculated using (5) for the coasting beam and (14) for the bunched beam only apply to a part of the particles. This tune spread serves as a convention, rather than a verifiable criterion, to estimate the space charge effect. In this sense, an unified calculation is important, as we have tried to address in this note.

- The use of second harmonic RF system can effectively reduce the beam peak current, and therefore the space charge tune spread. At the bunching factor of 0.4, with the voltage of the second harmonic RF being set at a half of the first harmonic RF voltage, the beam peak current, and therefore the tune spread, can be reduced by as much as $25 \%$.

- In an $H^{-}$charge exchange injection, one may also project particles in the transverse phase space as 'smolke ring', and perform 'painting'. This has two advantages in terms of reducing the tune spread. Firstly, it gives rise to somewhat transverse flatop beam current, analogous to the longitudinal flatop line density created by the second harmonic RF system. Secondly, in a 'smoke ring' distribution, the particles in the phase space center are missing, and these particles have small oscillation amplitudes and presumably large tune shifts. Therefore, the space charge tune spread is further reduced. Using the 'smoke ring' distribution, the advantages associated with the large tune spread in terms of the beam transverse instabilities have been, of course, compromised.

- There are still other factors, which might affect the tune spread. These include i) the potential well effect through the change in the particle distribution in longitudinal phase space, ii) the sextupole and octupole effects, by momentum and oscillation amplitude dependent tune shifts, respectively, and iii) The use of debunchers in the Linac transfer

line to manipulate the momentum distribution, and therefore affects the bunch shape in the synchrotron. 


\section{References}

[1] B. Zotter, CERN 85-19, p.253, 1985.

[2] P.J. Bryant, CERN 87-10, p.62, 1987.

[3] A. Hofmann, CERN 94-01, p.329, 1994.

[4] L.J. Laslett, Proc. of 1963 summer Study of Storage Rings, BNL-Report 7534, p. 324, 1963.

[5] A. Chao, Physics of Collective Beam Instabilities in High Energy Accelerators, Wiley, New York, 1994.

[6] J.P. Delahaye et al. Proc. 11th Int. Conf. High Energy Acc. p.299, 1980.

[7] G. Guignard, CERN 77-10, 1977.

[8] F. Sacherer, IEEE Trans. Nucl. Sci., Vol.NS-24, p.1393, 1977. 